PROCEEDINGS OF THE

AMERICAN MATHEMATICAL SOCIETY

Volume 129, Number 6, Pages 1705-1711

S 0002-9939(00)05750-6

Article electronically published on October 31, 2000

\title{
A LIFTING THEOREM FOR SYMMETRIC COMMUTANTS
}

\author{
GELU POPESCU
}

(Communicated by David R. Larson)

\begin{abstract}
Let $T_{1}, \ldots, T_{n} \in B(\mathcal{H})$ be bounded operators on a Hilbert space $\mathcal{H}$ such that $T_{1} T_{1}^{*}+\cdots+T_{n} T_{n}^{*} \leq I_{\mathcal{H}}$. Given a symmetry $j$ on $\mathcal{H}$, i.e., $j^{2}=j^{*} j=I_{\mathcal{H}}$, we define the $j$-symmetric commutant of $\left\{T_{1}, \ldots, T_{n}\right\}$ to be the operator space

$$
\left\{A \in B(\mathcal{H}): T_{i} A=j A T_{i}, i=1, \ldots, n\right\} .
$$

In this paper we obtain lifting theorems for symmetric commutants. The result extends the Sz.-Nagy-Foias commutant lifting theorem $\left(n=1, j=I_{\mathcal{H}}\right)$, the anticommutant lifting theorem of Sebestyén $\left(n=1, j=-I_{\mathcal{H}}\right)$, and the noncommutative commutant lifting theorem $\left(j=I_{\mathcal{H}}\right)$. Sarason's interpolation theorem for $H^{\infty}$ is extended to symmetric commutants on Fock spaces.
\end{abstract}

\section{LIFTING THEOREM FOR SYMMETRIC COMMUTANTS}

Let $\mathbb{F}_{n}^{+}$be the unital free semigroup on $n$ generators $s_{1}, \ldots, s_{n}$, and let $e$ be its neutral element. For any $\sigma:=s_{i_{1}} \cdots s_{i_{k}} \in \mathbb{F}_{n}^{+}$, we define its length $|\sigma|:=k$, and $|e|=0$. On the other hand, if $T_{i} \in B(\mathcal{H}), i=1, \ldots, n$, we denote $T_{\sigma}:=T_{i_{1}} \cdots T_{i_{k}}$ and $T_{e}:=I_{\mathcal{H}}$.

Let us recall from [Po1], [Po2], and [Po4] a few results concerning the noncommutative dilation theory for $n$-tuples of operators. A sequence of operators $\mathcal{T}:=\left[T_{1}, \ldots, T_{n}\right], T_{i} \in B(\mathcal{H}), i=1, \ldots, n$, is called contractive (or row contraction) if $T_{1} T_{1}^{*}+\cdots+T_{n} T_{n}^{*} \leq I_{\mathcal{H}}$. We say that a sequence of isometries $\mathcal{V}:=\left[V_{1}, \ldots, V_{n}\right]$ on a Hilbert space $\mathcal{K} \supset \mathcal{H}$ is a minimal isometric dilation of $\mathcal{T}$ if the following properties are satisfied:

(i) $V_{1} V_{1}^{*}+\cdots+V_{n} V_{n}^{*} \leq I_{\mathcal{K}}$;

(ii) $\left.V_{i}^{*}\right|_{\mathcal{H}}=T_{i}^{*}, i=1, \ldots, n$;

(iii) $\mathcal{K}=\bigvee_{\alpha \in \mathbb{F}_{n}^{+}} V_{\alpha} \mathcal{H}$.

Consider the full Fock space on $n$ generators

$$
F^{2}\left(H_{n}\right):=\mathbb{C} 1 \oplus \bigoplus_{m \geq 1} H_{n}^{\otimes m},
$$

where $H_{n}$ is an $n$-dimensional complex Hilbert space with orthonormal basis $\left\{e_{1}, e_{2}, \ldots, e_{n}\right\}$ if $n$ is finite, and $\left\{e_{1}, e_{2}, \ldots\right\}$ if $n=\infty$. For each $i=1,2, \ldots$, let $S_{i}$ be the left creation operator with $e_{i}$, i.e.,

$$
S_{i} \xi:=e_{i} \otimes \xi, \quad \xi \in F^{2}\left(H_{n}\right) .
$$

Received by the editors March 1, 1999 and, in revised form, September 16, 1999.

2000 Mathematics Subject Classification. Primary 47F25, 47A57, 47A20; Secondary 30E05.

The author was partially supported by NSF Grant DMS-9531954. 
As in [Po1], let us define $D_{\mathcal{T}}: \bigoplus_{j=1}^{n} \mathcal{H} \rightarrow \bigoplus_{j=1}^{n} \mathcal{H}$ by $D_{\mathcal{T}}:=\left(I_{\oplus_{j=1}^{n}} \mathcal{H}-\mathcal{T}^{*} \mathcal{T}\right)^{1 / 2}$, and set $\mathcal{D}:=\overline{D_{\mathcal{T}}\left(\bigoplus_{j=1}^{n} \mathcal{H}\right)}$. Let $D_{i}: \mathcal{H} \rightarrow F^{2}\left(H_{n}\right) \otimes \mathcal{D}$ be defined by

$$
D_{i} h:=1 \otimes D_{\mathcal{T}}(\underbrace{0, \ldots, 0}_{i-1 \text { times }}, h, 0, \ldots, 0) \oplus 0 \oplus 0 \cdots .
$$

Consider the Hilbert space $\mathcal{K}:=\mathcal{H} \oplus\left(F^{2}\left(H_{n}\right) \otimes \mathcal{D}\right)$ and define $V_{i}: \mathcal{K} \rightarrow \mathcal{K}$ by

$$
V_{i}(h \oplus(\xi \otimes d)):=T_{i} h \oplus\left(D_{i} h+\left(S_{i} \otimes I_{\mathcal{D}}\right)(\xi \otimes d)\right)
$$

for any $h \in \mathcal{H}, \xi \in F^{2}\left(H_{n}\right), d \in \mathcal{D}$. Notice that

$$
V_{i}=\left[\begin{array}{cc}
T_{i} & 0 \\
D_{i} & S_{i} \otimes I_{\mathcal{D}}
\end{array}\right]
$$

with respect to the decomposition $\mathcal{K}=\mathcal{H} \oplus\left(F^{2}\left(H_{n}\right) \otimes \mathcal{D}\right)$. It was proved in Po1] that the sequence $\mathcal{V}:=\left[V_{1}, \ldots, V_{n}\right]$ is the minimal isometric dilation of $\mathcal{T}$, and it is uniquely determined up to an isomorphism. Let $\mathcal{H}_{0}:=\mathcal{H}$ and

$$
\mathcal{H}_{k}:=\mathcal{H}_{k-1} \vee\left(\bigvee_{|\alpha|=1} V_{\alpha} \mathcal{H}_{k-1}\right) \quad \text { if } k \geq 1
$$

Notice that $\mathcal{K}=\bigvee_{k=0}^{\infty} \mathcal{H}_{k}, \mathcal{H}_{k} \subseteq \mathcal{H}_{k+1}$, and all subspaces $\mathcal{H}_{k}$ are invariant to each $V_{i}^{*}, i=1, \ldots, n$. On the other hand, we have $\mathcal{H}_{1}=\mathcal{H} \oplus \mathcal{D}$ and

$$
\mathcal{H}_{k}=\mathcal{H} \oplus \bigoplus_{|\alpha| \leq k-1} e_{\alpha} \otimes \mathcal{D} \quad \text { if } k \geq 2
$$

where $\left\{e_{\alpha}\right\}_{\alpha \in \mathbb{F}_{n}^{+}}$is the canonical basis of $F^{2}\left(H_{n}\right)$ generated by $e_{1}, \ldots, e_{n}$, i.e., $e_{\alpha}:=$ $e_{i_{1}} \otimes \cdots \otimes e_{i_{k}}$ if $\alpha:=s_{i_{1}} \cdots s_{i_{k}} \in \mathbb{F}_{n}^{+}$, and $e_{\alpha}=1$ if $\alpha=e$. Denote by $P_{\mathcal{H}_{k}}$ the orthogonal projection from $\mathcal{K}$ onto $\mathcal{H}_{k}$, and notice that

$$
P_{\mathcal{H}_{k+1}} V_{i}=V_{i} P_{\mathcal{H}_{k}} \text { for any } i=1, \ldots, n,
$$

and $k=0,1, \ldots$

Let us recall from [Se the following consequence of Krein's self-adjoint extension theorem [K].

Lemma 1.1. Let $\mathcal{M}, \mathcal{M}^{\prime}$ be Hilbert spaces, $\mathcal{N} \subseteq \mathcal{M}, \mathcal{N}^{\prime} \subseteq \mathcal{M}^{\prime}$ be subspaces, and $X \in B\left(\mathcal{N}, \mathcal{M}^{\prime}\right), X^{\prime} \in B\left(\mathcal{N}^{\prime}, \mathcal{M}\right)$ be bounded operators. Then there exists $Y \in B\left(\mathcal{M}, \mathcal{M}^{\prime}\right)$ such that $\left.Y\right|_{\mathcal{N}}=X$ and $\left.Y^{*}\right|_{\mathcal{N}^{\prime}}=X^{\prime}$ if and only if

$$
\left\langle X h, h^{\prime}\right\rangle=\left\langle h, X^{\prime} h^{\prime}\right\rangle
$$

for any $h \in \mathcal{N}, h^{\prime} \in \mathcal{N}$. Moreover, $Y$ can be chosen of norm $\max \left\{\|X\|,\left\|X^{\prime}\right\|\right\}$.

Proof. Let $A: \mathcal{N} \oplus \mathcal{N}^{\prime} \rightarrow \mathcal{M} \oplus \mathcal{M}^{\prime}$ be defined by

$$
\left[\begin{array}{cc}
0 & X^{\prime} \\
X & 0
\end{array}\right]
$$

and notice that $\|A\|=\max \left\{\|X\|,\left\|X^{\prime}\right\|\right\}$. Since (1.3) holds, $A$ is a symmetric operator, i.e., $\langle A x, y\rangle=\langle x, A y\rangle$ for any $x, y \in \mathcal{N} \oplus \mathcal{N}^{\prime}$. According to Krein's selfadjoint extension theorem $\left[\mathrm{K}\right.$, there exists $B \in B\left(\mathcal{M} \oplus \mathcal{M}^{\prime}\right)$ such that $B=B^{*}$, $\left.B\right|_{\mathcal{N} \oplus \mathcal{N}^{\prime}}=A$, and $\|B\|=\|A\|$. The operator $Y \in B\left(\mathcal{M}, \mathcal{M}^{\prime}\right)$ defined by $Y:=$ $\left.P_{\mathcal{M}^{\prime}} B\right|_{\mathcal{M}}$, where $P_{\mathcal{M}^{\prime}}$ is the orthogonal projection from $\mathcal{M} \oplus \mathcal{M}^{\prime}$ to $\mathcal{M}^{\prime}$, has the required properties. 
Let $\mathcal{T}:=\left[T_{1}, \ldots, T_{n}\right], T_{i} \in B(\mathcal{H})$, be a row contraction, $\mathcal{V}:=\left[V_{1}, \ldots, V_{n}\right]$ be its minimal isometric dilation on $\mathcal{K} \supseteq \mathcal{H}$, and $\mathcal{H}_{k} \subseteq \mathcal{K}, k=0,1, \ldots$, be defined by (1.1). Let $j \in B(\mathcal{H})$ be a symmetry on $\mathcal{H}$, i.e., $j^{2}=j^{*} j=I_{\mathcal{H}}$.

We say that $A \in B(\mathcal{H})$ is in the $j$-commutant of $\left\{T_{1}, \ldots, T_{n}\right\}$ if and only if

$$
T_{i} A=j A T_{i} \text { for any } i=1, \ldots, n .
$$

The main result of this paper is the following lifting theorem for $j$-commutants.

Theorem 1.2. Let $\mathcal{T}:=\left[T_{1}, \ldots, T_{n}\right]$ be a row contraction with $T_{i} \in B(\mathcal{H})$ and let $\mathcal{V}:=\left[V_{1}, \ldots, V_{n}\right]$ be its minimal isometric dilation on $\mathcal{K} \supseteq \mathcal{H}$. Let $j$ be a symmetry on $\mathcal{H}$ and let $J \in B(\mathcal{K})$ be a contraction such that each $\mathcal{H}_{k}, k=0,1, \ldots$, reduces $J$ and $\left.J\right|_{\mathcal{H}}=j$. If $A \in B(\mathcal{H})$ is in the $j$-commutant of $\left\{T_{1}, \ldots, T_{n}\right\}$, then there exists $A_{J} \in B(\mathcal{K})$ in the $J$-commutant of $\left\{V_{1}, \ldots, V_{n}\right\}$ such that

$$
\left.A_{J}^{*}\right|_{\mathcal{H}}=A^{*} \text { and }\left\|A_{J}\right\|=\|A\| .
$$

Proof. We use the notation and preliminaries preceding the theorem. Our goal is to construct a sequence $A_{k} \in B\left(\mathcal{H}_{k}\right), k=1,2, \ldots$, with the following properties:

(i) $\left.A_{k}^{*}\right|_{\mathcal{H}_{k-1}}=A_{k-1}^{*}$;

(ii) $\left.A_{k}\right|_{\bigvee_{i=1}^{n} V_{i} \mathcal{H}_{k-1}}=\left.\left(\sum_{i=1}^{n} P_{\mathcal{H}_{k}} J V_{i} A_{k-1} V_{i}^{*}\right)\right|_{\bigvee_{i=1}^{n} V_{i} \mathcal{H}_{k-1}}$;

(iii) $P_{\mathcal{H}_{k}} J V_{i} A_{k-1} P_{\mathcal{H}_{k-1}}=A_{k} P_{\mathcal{H}_{k}} V_{i}, i=1, \ldots, n$;

(iv) $\left\|A_{k}\right\|=\left\|A_{k-1}\right\|=\|A\|$.

Once this is established, since $\mathcal{K}=\bigvee_{k=0}^{\infty} \mathcal{H}_{k}$, it will be easy to see that the limit $A_{J}^{*}:=\mathrm{SOT}-\lim _{k \rightarrow \infty} A_{k}^{*} P_{\mathcal{H}_{k}}$ exists and $A_{J}$ has the stated properties in the theorem.

Our first step is to show that $A_{1}$ exists with the above-mentioned properties, when $k=1$. Since $P_{\mathcal{H}} J=J P_{\mathcal{H}},\left.J\right|_{\mathcal{H}}=j, A \in B(\mathcal{H})$ is in the $j$-commutant of $\left\{T_{1}, \ldots, T_{n}\right\}, j^{2}=1$, and $\mathcal{V}$ is the minimal isometric dilation of $\mathcal{T}$, we have

$$
\begin{aligned}
\left\langle\left(\sum_{i=1}^{n} P_{\mathcal{H}_{1}} J V_{i} A V_{i}^{*}\right)\left(\sum_{i=1}^{n} V_{i} h_{i}\right), h\right\rangle & =\left\langle\sum_{i=1}^{n} P_{\mathcal{H}} J V_{i} A h_{i}, h\right\rangle=\left\langle\sum_{i=1}^{n} j T_{i} A h_{i}, h\right\rangle \\
& =\left\langle\sum_{i=1}^{n} j^{2} A T_{i} h_{i}, h\right\rangle=\left\langle\sum_{i=1}^{n} P_{\mathcal{H}} T_{i} h_{i}, A^{*} h\right\rangle \\
& =\left\langle\sum_{i=1}^{n} V_{i} h_{i}, A^{*} h\right\rangle
\end{aligned}
$$

for any $h_{i}, h \in \mathcal{H}$. On the other hand, since $\|J\| \leq 1, P_{\mathcal{H}_{1}} J=J P_{\mathcal{H}_{1}}$, and $V_{1}, \ldots, V_{n}$ have orthogonal ranges, we have

$$
\begin{aligned}
\left\|\left(\sum_{i=1}^{n} P_{\mathcal{H}_{1}} J V_{i} A V_{i}^{*}\right)\left(\sum_{i=1}^{n} V_{i} h_{i}\right)\right\|^{2} & =\left\|\sum_{i=1}^{n} J V_{i} A h_{i}\right\|^{2} \\
& \leq\left\|\sum_{i=1}^{n} V_{i} A h_{i}\right\|^{2}=\sum_{i=1}^{n}\left\|A h_{i}\right\|^{2} \\
& \leq\|A\|^{2}\left\|\sum_{i=1}^{n} V_{i} h_{i}\right\|^{2}
\end{aligned}
$$


Therefore,

$$
\left\|\left.\left(\sum_{i=1}^{n} P_{\mathcal{H}_{1}} J V_{i} A V_{i}^{*}\right)\right|_{\vee_{i=1}^{n} V_{i} \mathcal{H}}\right\| \leq\|A\| .
$$

Now, we apply Lemma 1.1 in the particular case when

$$
\begin{aligned}
& \mathcal{M}:=\mathcal{M}^{\prime}:=\mathcal{H}_{1}:=\mathcal{H} \vee \bigvee_{i=1}^{n} V_{i} \mathcal{H} \\
& \mathcal{N}:=\bigvee_{i=1}^{n} V_{i} \mathcal{H}, \quad \mathcal{N}^{\prime}:=\mathcal{H}
\end{aligned}
$$

and $X \in B\left(\mathcal{N}, \mathcal{M}^{\prime}\right)$ is defined by $X:=\left.\left(\sum_{i=1}^{n} P_{\mathcal{H}_{1}} J V_{i} A V_{i}^{*}\right)\right|_{\bigvee_{i=1}^{n} V_{i} \mathcal{H}}$, and $X^{\prime} \in$ $B\left(\mathcal{N}^{\prime}, \mathcal{M}\right)$ by $X^{\prime}:=A^{*}$. Therefore, we find $A_{1} \in B\left(\mathcal{H}_{1}\right)$ such that $\left.A_{1}^{*}\right|_{\mathcal{H}}=A^{*}$, $\left\|A_{1}\right\|=\|A\|$, and $\left.A_{1}\right|_{\bigvee_{i=1}^{n} V_{i} \mathcal{H}}=\left.\left(\sum_{i=1}^{n} P_{\mathcal{H}_{1}} J V_{i} A V_{i}^{*}\right)\right|_{\bigvee_{i=1}^{n} V_{i} \mathcal{H}}$. On the other hand, since $V_{i} P_{\mathcal{H}}=P_{\mathcal{H}_{1}} V_{i}$ and $V_{p}^{*} V_{i}=\delta_{p i} I_{\mathcal{K}}$, we infer that

$$
\begin{aligned}
P_{\mathcal{H}_{1}} J V_{i} A P_{\mathcal{H}} & =\left(\sum_{p=1}^{n} P_{\mathcal{H}_{1}} J V_{p} A V_{p}^{*}\right) V_{i} P_{\mathcal{H}} \\
& =A_{1} V_{i} P_{\mathcal{H}}=A_{1} P_{\mathcal{H}_{1}} V_{i}
\end{aligned}
$$

for any $i=1, \ldots, n$.

Our second step is to apply again Lemma 1.1 in order to to find $A_{2} \in B\left(\mathcal{H}_{2}\right)$ such that $\left.A_{2}^{*}\right|_{\mathcal{H}_{1}}=A_{1}^{*},\left\|A_{2}\right\|=\left\|A_{1}\right\|,\left.A_{2}\right|_{\bigvee_{i=1}^{n} V_{i} \mathcal{H}_{1}}=\left.\left(\sum_{i=1}^{n} P_{\mathcal{H}_{2}} J V_{i} A_{1} V_{i}^{*}\right)\right|_{\bigvee_{i=1}^{n} V_{i} \mathcal{H}_{1}}$, and, moreover,

$$
P_{\mathcal{H}_{1}} J V_{i} A P_{\mathcal{H}}=A_{1} P_{\mathcal{H}_{1}} V_{i}
$$

for any $i=1, \ldots, n$. Let $k, k_{i}, i=1, \ldots, n$, be in $\mathcal{H}_{1}$. Taking into account that $J P_{\mathcal{H}_{1}}=P_{\mathcal{H}_{1}} J, P_{\mathcal{H}_{1}} V_{i}=V_{i} P_{\mathcal{H}}, P_{\mathcal{H}} A_{1}=A P_{\mathcal{H}}$, and the relation (1.4), we infer the following

$$
\begin{aligned}
\left\langle\left(\sum_{i=1}^{n} P_{\mathcal{H}_{2}} J V_{i} A_{1} V_{i}^{*}\right)\left(\sum_{i=1}^{n} V_{i} k_{i}\right), k\right\rangle & =\left\langle\sum_{i=1}^{n} P_{\mathcal{H}_{2}} J V_{i} A_{1} k_{i}, k\right\rangle=\left\langle\sum_{i=1}^{n} J P_{\mathcal{H}_{1}} V_{i} A_{1} k_{i}, k\right\rangle \\
& =\left\langle\sum_{i=1}^{n} J V_{i} P_{\mathcal{H}} A_{1} k_{i}, k\right\rangle=\left\langle\sum_{i=1}^{n} P_{\mathcal{H}_{1}} J V_{i} A P_{\mathcal{H}} k_{i}, k\right\rangle \\
& =\left\langle\sum_{i=1}^{n} A_{1} P_{\mathcal{H}_{1}} V_{i} k_{i}, k\right\rangle=\left\langle\sum_{i=1}^{n} V_{i} k_{i}, A_{1}^{*} k\right\rangle .
\end{aligned}
$$

On the other hand, since $\|J\| \leq 1, P_{\mathcal{H}_{2}} J=J P_{\mathcal{H}_{2}}$, and $V_{1}, \ldots, V_{n}$ have orthogonal ranges, we infer that

$$
\left\|\left.\left(\sum_{i=1}^{n} P_{\mathcal{H}_{2}} J V_{i} A_{1} V_{i}^{*}\right)\right|_{\bigvee_{i=1}^{n} V_{i} \mathcal{H}_{1}}\right\| \leq\left\|A_{1}\right\|
$$

(see the first step for similar computations). 
Using Lemma 1.1, we find $A_{2}$ with the above-mentioned properties. On the other hand, since $V_{i} P_{\mathcal{H}_{1}}=P_{\mathcal{H}_{2}} V_{i}$ and $V_{p}^{*} V_{i}=\delta_{p i} I_{\mathcal{K}}$, we infer that

$$
\begin{aligned}
P_{\mathcal{H}_{2}} J V_{i} A_{1} P_{\mathcal{H}_{1}} & =\left(\sum_{p=1}^{n} P_{\mathcal{H}_{2}} J V_{p} A V_{p}^{*}\right) V_{i} P_{\mathcal{H}_{1}} \\
& =A_{2} V_{i} P_{\mathcal{H}_{1}}=A_{1} P_{\mathcal{H}_{2}} V_{i}
\end{aligned}
$$

for any $i=1, \ldots, n$.

Iterating the process, we obtain at the $k$ th step an operator $A_{k} \in B\left(\mathcal{H}_{k}\right)$ satisfying the properties (i), (ii), (iii), (iv). The proof is complete.

Let us remark that in the particular case when $n=1, j=I_{\mathcal{H}}$, and $J=I_{\mathcal{K}}$, we find again the Sz.-Nagy-Foiaş commutant lifting theorem [SzF1, SzF2] (see also S], [DMP]). The anticommutant lifting theorem of Sebestyén [Se] is obtained when $n=1, j=-I_{\mathcal{H}}$, and $J=-I_{\mathcal{K}}$. When $j=I_{\mathcal{H}}$ and $J=I_{\mathcal{K}}$, we obtain a new proof of the noncommutative commutant lifting theorem [Po4]. We refer to [Po5], Po7], [Po8], [Po9], and [APo, for applications of this theorem to interpolation in several variables.

\section{Symmetric Commutants on Fock spaces}

For each $i=1,2, \ldots$, let $S_{i}$ be the left creation operator with $e_{i}$, i.e.,

$$
S_{i} \xi:=e_{i} \otimes \xi, \xi \in F^{2}\left(H_{n}\right) .
$$

We shall denote by $\mathcal{P}$ the set of all $p \in F^{2}\left(H_{n}\right)$ which are sums of finite number of tensor monomials, i.e., $p=a_{0}+\sum a_{i_{1} \cdots i_{k}} e_{i_{1}} \otimes \cdots \otimes e_{i_{k}}$, where $a_{0}, a_{i_{1} \ldots i_{k}} \in \mathbb{C}$. The set $\mathcal{P}$ may be viewed as the algebra of polynomials in $n$ noncommuting indeterminates, with $p \otimes q, p, q \in \mathcal{P}$, as multiplication. Define $F_{n}^{\infty}$ as the set of all $g \in F^{2}\left(H_{n}\right)$ such that

$$
\|g\|_{\infty}:=\sup \left\{\|g \otimes p\|_{2}: p \in \mathcal{P},\|p\|_{2} \leq 1\right\}<\infty
$$

where $\|\cdot\|_{2}:=\|\cdot\|_{F^{2}\left(H_{n}\right)}$. The Banach algebra $F_{n}^{\infty}$ can be viewed as a noncommutative analogue of the Hardy space $H^{\infty}$; when $n=1$ they coincide. It follows from Po6 that the noncommutative analytic Toeplitz algebra $F_{n}^{\infty}$ can be identified with the WOT-closed algebra generated by the left creation operators $S_{1}, \ldots S_{n}$, and the identity. The algebra $F_{n}^{\infty}$ was introduced by the author in $\mathrm{Po} 3$ in connection with a noncommutative von Neumann type inequality in several variables. In particular, we proved that there is a completely contractive homomorphism

$$
\Phi: F_{n}^{\infty} \rightarrow H^{\infty}\left(\mathbb{B}_{n}\right), \quad[\Phi(f)]\left(\lambda_{1}, \ldots, \lambda_{n}\right)=f\left(\lambda_{1}, \ldots, \lambda_{n}\right),
$$

where $f:=f\left(S_{1}, \ldots, S_{n}\right) \in F_{n}^{\infty}$ and $\left(\lambda_{1}, \ldots, \lambda_{n}\right) \in \mathbb{B}_{n}$, the open unit ball of $\mathbb{C}^{n}$. This homomorphism established a strong connection between the algebra $F_{n}^{\infty}$ and the function theory of the open unit ball $\mathbb{B}^{n}$ (see $\mathrm{APO},[\mathrm{Po} 7$ ).

We recall from $\mathrm{Po}$ ] the characterization of the commutant of $\left\{S_{1}, \ldots, S_{n}\right\}$. Define the flipping operator $U: F^{2}\left(H_{n}\right) \rightarrow F^{2}\left(H_{n}\right)$ by

$$
U\left(e_{i_{1}} \otimes e_{i_{2}} \otimes \cdots \otimes e_{i_{k}}\right)=e_{i_{k}} \otimes \cdots \otimes e_{i_{2}} \otimes e_{i_{1}},
$$

and let $\tilde{\varphi}:=U \varphi$. It is easy to see that $U$ is a unitary operator, which satisfies $U(\varphi \otimes \psi)=\tilde{\psi} \otimes \tilde{\varphi}$, and $U^{2}=I$. According to Theorem 1.2 from [Po5], an operator $A \in B\left(F^{2}\left(H_{n}\right)\right)$ commutes with $\left\{S_{1}, \ldots, S_{n}\right\}$ if and only if there exists $\phi \in F_{n}^{\infty}$ such that $A h=h \otimes \tilde{\phi}, h \in F^{2}\left(H_{n}\right)$. Notice that $A=U^{*} \phi\left(S_{1}, \ldots, S_{n}\right) U$, where 
$\phi\left(S_{1}, \ldots, S_{n}\right)$ is the left multiplication operator by $\phi$ on the Fock space $F^{2}\left(H_{n}\right)$. In what follows we use the natural identification of $\phi$ with $\phi\left(S_{1}, \ldots, S_{n}\right)$.

As in [Po2], we say that an element $\varphi \in F_{n}^{\infty}$ is inner if $\varphi$ is an isometry, and outer if it has dense range. A family of inner elements $\left\{\varphi_{i}: i \in J\right\}$ is called orthogonal if $\varphi_{i}$ and $\varphi_{j}$ have orthogonal ranges, whenever $i \neq j$. A complete description of the invariant subspace structure of $F_{n}^{\infty}$ was obtained in [Po2] (even in a more general setting), using a noncommutative version of the Wold decomposition (see [Po1]). It follows from Theorem 2.2 of $\mathrm{Po} 2$ that a subspace $\mathcal{N}$ of $F^{2}\left(H_{n}\right)$ is invariant under $S_{1}, \ldots, S_{n}$ if and only if

$$
\mathcal{N}=\bigoplus_{i \in J} U^{*} \varphi_{i} U\left[F^{2}\left(H_{n}\right)\right]
$$

for some family $\left\{\varphi_{i}: i \in J\right\}$ of orthogonal inner elements (see also [Po5]).

In what follows we extend Sarason's result $[\mathrm{S}]$ to symmetric commutants on Fock spaces. Let $\mathcal{K}$ be a Hilbert space and let $F_{n}^{\infty} \bar{\otimes} B(\mathcal{K})$ be the WOT-closed algebra generated by the spatial tensor product of the two algebras.

Theorem 2.1. Let $j \in B(\mathcal{K})$ be a symmetry on a Hilbert space $\mathcal{K}$ and let $\mathcal{N} \subseteq$ $F^{2}\left(H_{n}\right)$ be an invariant subspace for each $S_{1}^{*}, \ldots, S_{n}^{*}$. If $X \in B(\mathcal{N} \otimes \mathcal{K})$ is in the $I_{\mathcal{N}} \otimes j$-commutant of $\left\{\left.P_{\mathcal{N} \otimes \mathcal{K}}\left(S_{i} \otimes I_{\mathcal{K}}\right)\right|_{\mathcal{N} \otimes \mathcal{K}}, i=1, \ldots, n\right\}$, then there exists $\Phi \in F_{n}^{\infty} \bar{\otimes} B(\mathcal{K})$ such that

$$
P_{\mathcal{N} \otimes \mathcal{K}} E\left(U^{*} \otimes I\right) \Phi(U \otimes I)=X P_{\mathcal{N} \otimes \mathcal{K}}, \quad\|\Phi\|=\|X\|,
$$

where $E \in B\left(F^{2}\left(H_{n}\right) \otimes \mathcal{K}\right)$ is the symmetry defined by

$$
E\left(\sum_{\alpha \in \mathbb{F}_{n}^{+}} e_{\alpha} \otimes k_{\alpha}\right):=\sum_{\alpha \in \mathbb{F}_{n}^{+}} e_{\alpha} \otimes j^{|\alpha|}\left(k_{\alpha}\right),
$$

$U$ is the flipping operator on $F^{2}\left(H_{n}\right)$, and $P_{\mathcal{N} \otimes \mathcal{K}}$ is the orthogonal projection of $F^{2}\left(H_{n}\right) \otimes \mathcal{K}$ onto $\mathcal{N} \otimes \mathcal{K}$.

Proof. Since $\left[\left.P_{\mathcal{N}} S_{1}\right|_{\mathcal{N}}, \ldots,\left.P_{\mathcal{N}} S_{n}\right|_{\mathcal{N}}\right]$ is a $C_{0}$-contraction, according to [Po1], its minimal isometric dilation is $\left[S_{1}, \ldots, S_{n}\right]$. Therefore, the minimal isometric dilation of $\left[\left.P_{\mathcal{N}} S_{1}\right|_{\mathcal{N}} \otimes I_{\mathcal{K}}, \ldots,\left.P_{\mathcal{N}} S_{n}\right|_{\mathcal{N}} \otimes I_{\mathcal{K}}\right]$ is $\left[S_{1} \otimes I_{\mathcal{K}}, \ldots, S_{n} \otimes I_{\mathcal{K}}\right]$. Since $I_{\mathcal{N}} \otimes j$ is a symmetry on $\mathcal{N} \otimes \mathcal{K}$, its extension $J:=I_{F^{2}\left(H_{n}\right)} \otimes j$ is a contraction on $F^{2}\left(H_{n}\right) \otimes \mathcal{K}$ satisfying the conditions of Theorem 1.2. Therefore, there exists

$$
A \in B\left(F^{2}\left(H_{n}\right) \otimes K\right)
$$

in the $I_{F^{2}\left(H_{n}\right)} \otimes j$-commutant of $\left\{S_{1} \otimes I_{\mathcal{K}}, \ldots, S_{n} \otimes I_{\mathcal{K}}\right\}$, i.e.,

$$
A\left(S_{i} \otimes I\right)=\left(I_{F^{2}\left(H_{n}\right)} \otimes j\right)\left(S_{i} \otimes I\right) A,
$$

such that

$$
P_{\mathcal{N} \otimes \mathcal{K}} A=X P_{\mathcal{N} \otimes \mathcal{K}} \text { and }\|A\|=\|X\| .
$$

Notice that the operator $E$ defined by $(2.2)$ is a symmetry on $F^{2}\left(H_{n}\right) \otimes K$ and

$$
E\left(S_{i} \otimes I_{\mathcal{K}}\right)=\left(I_{F^{2}\left(H_{n}\right)} \otimes j\right)\left(S_{i} \otimes I\right) E .
$$

Using (2.3) and (2.5), we infer that

$$
\begin{aligned}
E A\left(S_{i} \otimes I_{\mathcal{K}}\right) & =E\left(I_{F^{2}\left(H_{n}\right)} \otimes j\right)\left(S_{i} \otimes I\right) A \\
& =E^{2}\left(S_{i} \otimes I\right) E A=\left(S_{i} \otimes I\right) E A .
\end{aligned}
$$


Therefore $E A$ is in the commutant of $\left\{S_{1} \otimes I_{\mathcal{K}}, \ldots, S_{n} \otimes I_{\mathcal{K}}\right\}$. According to Po7, there exists $\Phi \in F^{\infty}\left(H_{n}\right) \bar{\otimes} B(\mathcal{K})$ such that $E A=\left(U^{*} \otimes I\right) \Phi(U \otimes I)$. Since $E$ is a symmetry, and using (2.4), the result follows.

Corollary 2.2. Let $j \in B(\mathcal{K})$ be a symmetry on a Hilbert space $\mathcal{K}$. Then the $I_{F^{2}\left(H_{n}\right)} \otimes j$-commutant of $\left\{S_{1} \otimes I_{\mathcal{K}}, \ldots, S_{n} \otimes I_{\mathcal{K}}\right\}$ is

$$
\left\{E\left(U^{*} \otimes I\right) \Phi(U \otimes I): \Phi \in F_{n}^{\infty} \bar{\otimes} B(\mathcal{K})\right\},
$$

where $E$ is the symmetry defined by (2.2).

Let us remark that Theorem 2.1 can be used to obtain versions of NevanlinnaPick type interpolation for $F_{n}^{\infty}$. Since the approach is similar to $\mathrm{APO}$, $\mathrm{Po}$, we leave this task to the reader. All the results of this paper hold true if we allow $n=\infty$.

\section{REFERENCES}

[APo] A. Arias and G. Popescu, Noncommutative interpolation and Poisson transforms, Israel J. Math. 115 (2000), 205-234. CMP 2000:10

[DMP] R.G. Douglas, P.S. Muhly and C. Pearcy, Lifting commuting operators, Michigan Math.J. 15 (1968), 385-395. MR 38:5046

[K] M.A. Krein, The theory of self-adjoint extensions of semi-bounded Hermitian transformations and its applications, Math. Collection, Moscow 20 (1947), 431-495. MR 9:515c

[Po1] G. Popescu, Isometric dilations for infinite sequences of noncommuting operators, Trans. Amer.Math.Soc. 316 (1989), 523-536. MR 90c:47006

[Po2] G. Popescu, Characteristic functions for infinite sequences of noncommuting operators, J.Operator Theory 22 (1989), 51-71. MR 91m:47012

[Po3] G. Popescu, Von Neumann inequality for $\left(B(H)^{n}\right)_{1}$, Math.Scand. 68 (1991), 292-304. MR 92k:47073

[Po4] G. Popescu, On intertwining dilations for sequences of noncommuting operators, J.Math. Anal.Appl. 167 (1992), 382-402. MR 93e:47012

[Po5] G. Popescu, Multi-analytic operators on Fock spaces, Math. Ann. 303 (1995), 31-46. MR 96k:47049

[Po6] G. Popescu, Functional calculus for noncommuting operators, Michigan Math. J. 42 (1995), 345-356. MR 96k:47025

[Po7] G. Popescu, Interpolation problems in several variables, J. Math Anal. Appl. 227 (1998), 227-250. MR 99i:47028

[Po8] G. Popescu, Commutant lifting, tensor algebras, and functional calculus, Proc. Edinburg Math. Soc. (2), to appear.

[Po9] G. Popescu, Spectral liftings in Banach algebras and interpolation in several variables, preprint, 1998.

[S] D. Sarason, Generalized interpolation in $H^{\infty}$, Trans. Amer. Math. Soc. 127 (1967), 179203. MR 34:8193

[Se] Z. Sebestyén, Anticommutant lifting and anticommuting dilation, Proc. Amer. Math. Soc. 121 (1994), 133-136. MR 94g:47009

[SzF1] B.Sz.-Nagy, C. Foiaş, Dilatation des commutants d'operateurs, C.R. Acad. Sci. Paris, Serie A 266 (1968), 493-495. MR 38:5049

[SzF2] B.Sz.-Nagy, C. Foiaş, Harmonic analysis on operators on Hilbert space, North-Holland, Amsterdam (1970). MR 43:947

Division of Mathematics and Statistics, The University of Texas at San Antonio, San Antonio, Texas 78249

E-mail address: gpopescu@math.utsa.edu 